\title{
Macro-Bending Influence on Radiation Induced Attenuation Measurement in Optical Fibres
}

\author{
Elisa Guillermain, Jochen Kuhnhenn, Daniel Ricci, and Udo Weinand
}

\begin{abstract}
Influence of the bending radius on the measurement of radiation induced attenuation in glass optical fibres is discussed in this paper. Radiation induced attenuation measured in two single-mode fibre types shows discrepancies when coiled around a low bending radius spool: the observed attenuation is lower than expected. A series of dedicated tests reveals that this invalid measurement is related to the displacement of the mode field towards the cladding when the fibre is bent with a low radius, and to the different radiation resistances of the core and cladding glasses. For irradiation tests of optical fibres, the spool radius should therefore be carefully chosen.
\end{abstract}

Index Terms-Bending radius, optical fibre, radiation effects, radiation induced attenuation (RIA).

\section{INTRODUCTION}

I $\mathrm{N}$ A harsh radioactive environment such as the CERN (European Organization for Nuclear Research) accelerator complex, optical fibres must be carefully chosen and qualified. Optical fibres to be installed at CERN are therefore tested for their radiation resistance. Their Radiation Induced Attenuation (RIA) is measured at the Fraunhofer INT irradiation facilities (Euskirchen, Germany).

Doses as high as $100 \mathrm{kGy}\left(\mathrm{SiO}_{2}\right)$ are expected each year in the most exposed areas (e.g. the collimation areas) of the Large Hadron Collider (LHC). Fibres intended to be installed in these areas are expected to resist at least up to this dose.

In the frame of a large procurement of specialty fibres, a quality assurance plan which includes the irradiation testing of all produced batches was followed, leading to about 65 sample tests. Reducing the fibre irradiation testing time is of interest under the point of view of cost and time management, thus tests at high dose rate are preferred. For a given point-like radioactive source, increasing the dose rate is achieved by getting closer to the source, i.e. using a low radius spool.

This paper focuses on the irradiation tests of telecom singlemode fibres compliant with the ITU-T G.652 international stan-

Manuscript received September 30, 2013; revised November 27, 2013; accepted February 13, 2014.

E. Guillermain and D. Ricci are with CERN, European Organization for Nuclear Research, CH-1211 Genève 23, Switzerland (e-mail: elisa.guillermain@cern.ch; daniel.ricci@cern.ch).

J. Kuhnhenn and U. Weinand are with Fraunhofer-Institut Naturwissenschaftlich Technische Trendanalysen (INT), Appelsgarten 2 D-53879, Euskirchen, Germany (e-mail: jochen.kuhnhenn@int.fraunhofer.de; udo.weinand@int.fraunhofer.de).

Color versions of one or more of the figures in this paper are available online at http://ieeexplore.ieee.org.

Digital Object Identifier 10.1109/TNS.2014.2306992

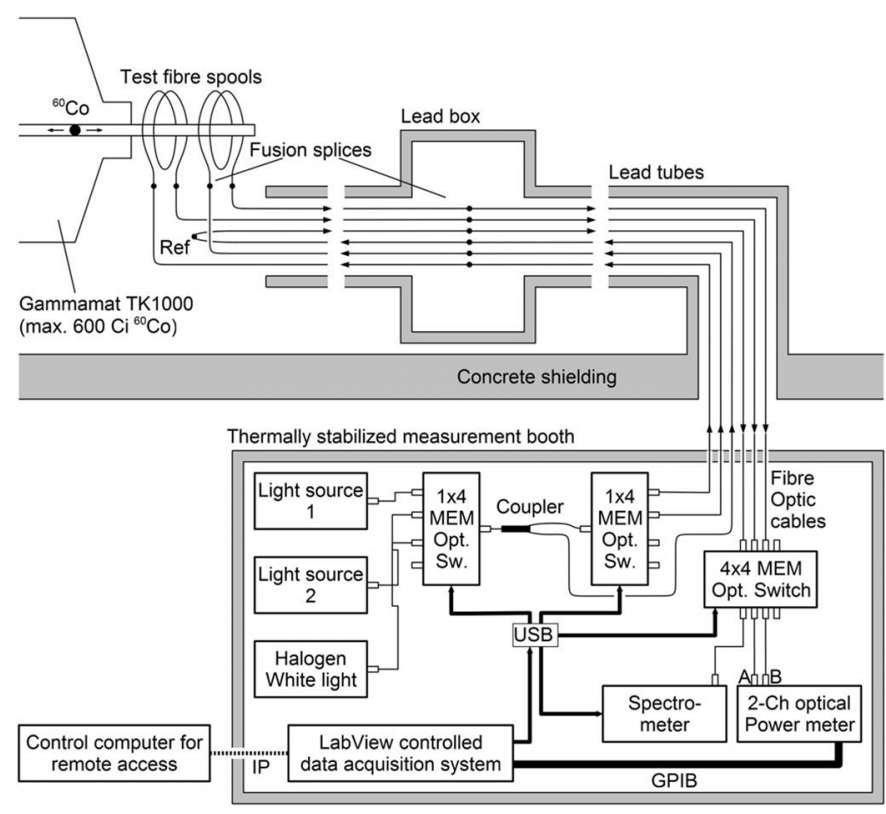

Fig. 1. Irradiation test setup for the simultaneous irradiation of two optical fibre samples. RIA is recorded at two wavelengths. A spectral analysis is also performed.

dard. The influence of the bending radius on their RIA is studied and discussed.

In this paper dose unit Gray (Gy) means $\mathrm{Gy}\left(\mathrm{SiO}_{2}\right)$.

\section{IRRADIATION TEST}

\section{A. Test Setup}

Optical fibre irradiation tests are performed with the setup shown in Fig. 1. Up to two fibres samples can be irradiated simultaneously, each of them coiled around an Aluminium spool of chosen radius and placed around a ${ }^{60} \mathrm{Co}$ source of known radioactivity. The distance of the sample from the source (i.e. the bending radius) determines the dose rate the fibre is exposed to.

Any mechanical stress or micro-bending (microscopic fibre stress or deformation) due to the spooling is suppressed before irradiation by relaxation of the coiled fibre. This relaxation is performed by carefully shaking the fibre spool with a specific apparatus involving acoustic waves [1]. The fibre sample is then checked with an OTDR (Optical Time-Domain Reflectometer) at $1310 \mathrm{~nm}, 1550 \mathrm{~nm}$, and $1625 \mathrm{~nm}$ to ensure it is stress free: no high attenuation was observed, even at longer wavelengths where the optical fibre is more sensitive to any kind of mechanical stress.

Before irradiation, the output light power is monitored in order to check the optical stability of the whole system (light 
sources, sample and detectors). When the setup stability is ensured, the ${ }^{60} \mathrm{Co}$ source is pushed out of the shielding container and starts irradiating the samples. The light power is then recorded as a function of time, for both discrete light sources (i.e. $1310 \mathrm{~nm}$ and $1570 \mathrm{~nm}$ ), alternatively in the tested fibres and in the reference fibre. In order to overcome any optical source drift during the test, the reference signal (in $\mathrm{dB}$ ) is afterwards subtracted from the tested fibre signal (in $\mathrm{dB}$ ). The resultant light power level is then divided by the optical fibre sample length. Thus the results are given in $\mathrm{dB} / \mathrm{km}$, avoiding disparate sample lengths to affect the results.

A spectral analysis is also performed by switching to the white light source and detecting light with the spectrometer, enabling to double-check the accuracy of the discrete-wavelength data.

Further details about the experimental equipment, sample preparation and uncertainty analysis can be found in [1].

\section{B. Testing Parameters}

Resistance to radiation of single-mode optical fibres is tested at both standard single-mode telecom operation wavelengths $(1310 \mathrm{~nm}$ and $1570 \mathrm{~nm}$ ). Light power is kept between $10 \mu \mathrm{W}$ and $40 \mu \mathrm{W}$ in order to minimize the effect of photobleaching [2].

The irradiation tests are performed at $1 \mathrm{~Gy} / \mathrm{s}$ and at $2 \mathrm{~Gy} / \mathrm{s}$. In the following, $1 \mathrm{~Gy} / \mathrm{s}$ tests are referred to as "low dose rate" tests and tests performed at a dose rate of $2 \mathrm{~Gy} / \mathrm{s}$ are referred to as "high dose rate" tests. The total dose reaches $75 \mathrm{kGy}$ or $110 \mathrm{kGy}$, depending on the dose rate.

To reach such dose rates, spools of $30 \mathrm{~mm}$ and of $18.5 \mathrm{~mm}$ radius were used. CERN specifies a minimum bending radius of $30 \mathrm{~mm}$ for installed fibres, thus a radius of $18.5 \mathrm{~mm}$ is below this specification.

All tests presented in this paper are performed at room temperature $\left(23 \pm 3^{\circ} \mathrm{C}\right)$.

\section{BENDING EFFECTS IN OPTICAL FIBRES}

The fibre under test needs to be bent (coiled) so that a significant length is exposed to the radiation. Such bending can induce macro-bending losses in the fibre (not to be mistaken with micro-bending losses that are due to microscopic fibre stress or deformation).

Macro-bending losses in an optical fibre are impacted by two factors: they increase with the number of turns around the spool (i.e. the bent fibre length) or by lowering the bending radius [3].

The cause of these macro-bending effects is related to the way light propagates in the fibre. In a single-mode fibre, light travels in both the core and the cladding: the mode field is distributed in the two regions. The mode field is displaced towards the cladding of the fibre when the fibre is bent [4]. The mode field displacement increases when lowering the bending radius and is eased if the mode field diameter (MFD) is large. Thus, because the MFD increases with the wavelength [5][6] the displacement of the mode field towards the cladding region is more significant at $1570 \mathrm{~nm}$ compared to $1310 \mathrm{~nm}$. Once a bending radius limit is reached (critical bending radius), the light escapes from the core and is not guided anymore.

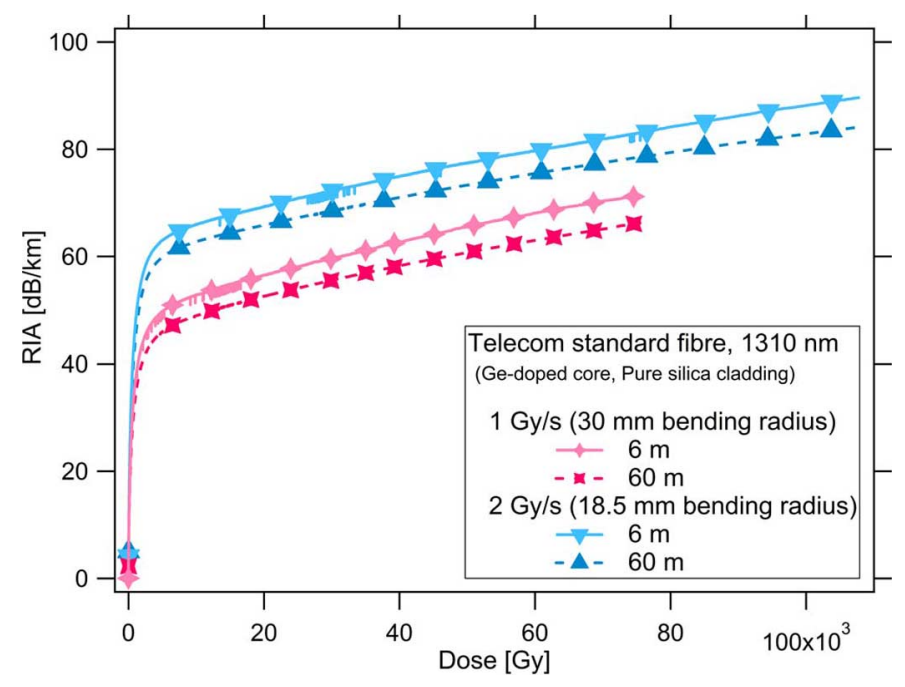

Fig. 2. Telecom standard fibre (Ge-doped core and pure silica cladding) RIA at $1310 \mathrm{~nm}$ for two different dose rates and sample lengths.

\section{IRRADIATION CONDITIONS}

A telecom standard single-mode optical fibre (ITU-T G.652.D) with Ge-doped core and pure silica cladding was selected for testing. According to the fibre manufacturer, 100 turns around a $25 \mathrm{~mm}$ radius spool or 1 turn around a $16 \mathrm{~mm}$ radius spool lead to bending losses that are below $0.05 \mathrm{~dB}$ for both $1310 \mathrm{~nm}$ and $1550 \mathrm{~nm}$. The fibre cutoff wavelength is equal to or below $1260 \mathrm{~nm}$.

Four optical fibre samples were irradiated at $1310 \mathrm{~nm}$ and $1570 \mathrm{~nm}$; two samples of $6 \mathrm{~m}$ length and two others of $60 \mathrm{~m}$ length. A $6 \mathrm{~m}$ sample and a $60 \mathrm{~m}$ sample were irradiated coiled around an $18.5 \mathrm{~mm}$ radius spool (52 and 520 turns respectively) to achieve a $2 \mathrm{~Gy} / \mathrm{s}$ dose rate. The two other samples were coiled around a $30 \mathrm{~mm}$ radius spool (32 and 320 turns respectively) leading to a $1 \mathrm{~Gy} / \mathrm{s}$ dose rate.

Although the high dose rate tests were performed with an unusually low bending radius, it is important to point out that no particularly high bending losses (macro-bending or micro-bending losses) were observed in the coiled samples before irradiation: OTDR measurements show that the samples were coiled above the critical bending radius and that they were stress-free even at $1625 \mathrm{~nm}$.

\section{RESULTS}

The obtained irradiation tests results at $1310 \mathrm{~nm}$ are presented in Fig. 2 and the $1570 \mathrm{~nm}$ results are presented in Fig. 3.

The results at $1 \mathrm{~Gy} / \mathrm{s}$ (samples coiled around a $30 \mathrm{~mm}$ radius spool) are shown for both wavelengths with star symbols. The $2 \mathrm{~Gy} / \mathrm{s}$ test results (samples coiled around a $18.5 \mathrm{~mm}$ radius spool) are shown with triangle symbols. The test results for the short $(6 \mathrm{~m})$ samples are shown with solid lines, whereas the test results for the long $(60 \mathrm{~m})$ samples are shown with dashed lines.

The RIA at same dose rate and same wavelength are expected to match regardless of the sample length. The RIA measured at high dose rate is expected to be higher than the RIA measured at low dose rate for a given wavelength [7]. Moreover, the specific single-mode fibre tested is known to exhibit higher RIA at $1310 \mathrm{~nm}$ than at $1570 \mathrm{~nm}$, with the RIA at $1570 \mathrm{~nm}$ becoming 


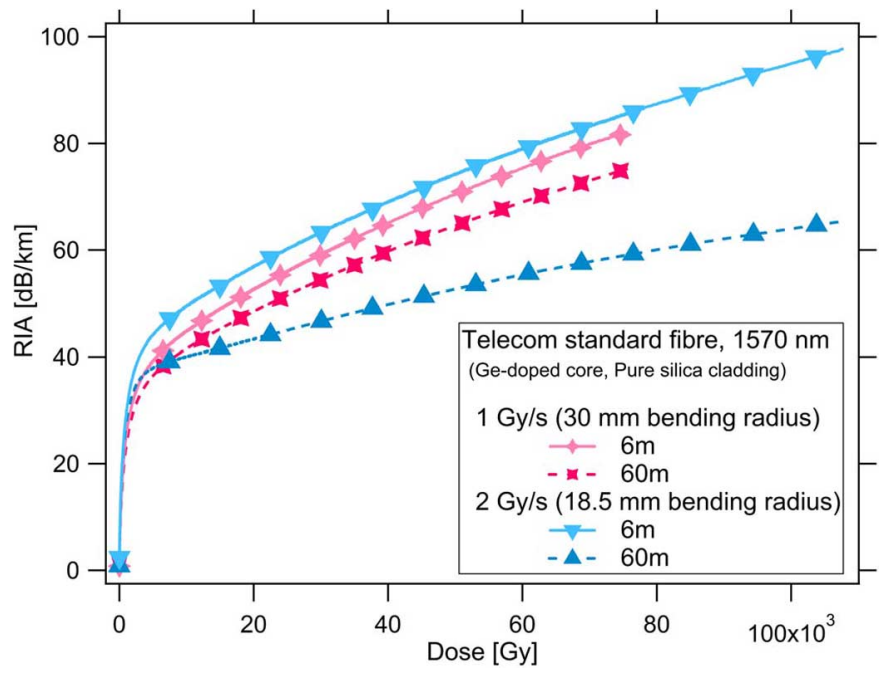

Fig. 3. Telecom standard fibre (Ge-doped core and pure silica cladding) RIA at $1570 \mathrm{~nm}$ for two different dose rates and sample lengths.

higher than the RIA at $1310 \mathrm{~nm}$ when the accumulated dose reaches a typical level (crossing point) that varies in function of the dose rate [8].

The irradiation test results at $1310 \mathrm{~nm}$ match the expected results: the RIA measured at $2 \mathrm{~Gy} / \mathrm{s}$ is higher than the RIA measured at $1 \mathrm{~Gy} / \mathrm{s}$, the difference being of about $12 \mathrm{~dB} / \mathrm{km}$ at a dose of $75 \mathrm{kGy}$. Hereinafter, an accumulated dose of $75 \mathrm{kGy}$ is implicitly assumed when talking about differences between RIA values. A small deviation is observed between the RIA values measured in the short sample or the long sample (about $5 \mathrm{~dB} / \mathrm{km}$ for both dose rates, which is significant compared to the uncertainties). This deviation is due to the difference between the actual dose rates to which the two samples are exposed. The samples being coiled with many turns and layers, the long length samples are exposed to an average dose rate that is slightly lower than the average dose rate to which the short samples are exposed. This is due to the fact that the outer coils of a long length sample are further from the radioactive source compared to the outer coils of a short length sample.

According to the irradiation test results at $1570 \mathrm{~nm}$ presented in Fig. 3, the RIA in the short $6 \mathrm{~m}$ sample is slightly higher than the RIA in the long $60 \mathrm{~m}$ sample (deviation of about $7 \mathrm{~dB} / \mathrm{km}$ ) when measured at $1 \mathrm{~Gy} / \mathrm{s}$. This behaviour is consistent with what is observed at $1310 \mathrm{~nm}$.

The RIA measured at $2 \mathrm{~Gy} / \mathrm{s}$ in the short $6 \mathrm{~m}$ sample is about $3.5 \mathrm{~dB} / \mathrm{km}$ higher than the RIA measured at $1 \mathrm{~Gy} / \mathrm{s}$, which appears to be consistent, even though a bigger difference could be expected comparing with the results at $1310 \mathrm{~nm}$. However, at high dose rate, the irradiation test results for the long length sample are quite different than what expected. The RIA in the long length sample should be higher at high dose rate than at low dose rate, but the opposite is observed: the RIA at $2 \mathrm{~Gy} / \mathrm{s}$ is $16 \mathrm{~dB} / \mathrm{km}$ lower than at $1 \mathrm{~Gy} / \mathrm{s}$.

These results clearly reveal an issue with the tests at $1570 \mathrm{~nm}$ and high dose rate (i.e. small spool radius) when using a long length sample.

\section{INTERPRETATION}

The short length samples show overall consistent RIA curves at both dose rates and wavelengths. However the long length sample measured RIA is totally unexpected when the sample is exposed to $2 \mathrm{~Gy} / \mathrm{s}$ and measured at $1570 \mathrm{~nm}$. Under these conditions the fibre exhibits an overall lower attenuation than anticipated.

The radiation resistance of the glass itself, in the material point of view, is influenced by the glass composition (dopant type and doping level), by the irradiation parameters (dose and dose rate) and depends on the wavelength, but is not influenced by any geometrical aspect, such as the fibre bending radius.

Because the measurement discrepancies are observed in conditions that are in favour of the mode field displacement towards the cladding (i.e. long wavelength, long fibre length and low bending radius), the proposed interpretation of the invalid RIA measurement is related to the mode field displacement and to the different radiation resistances of the core and cladding glasses. Due to the differences in glass compositions (i.e. different dopants and/or different doping levels), the core and the cladding are not affected in the same way when exposed to radiations [6], [9], [10]. When the fibre is bent, a larger amount of light travels in the cladding region because of the mode field displacement; however, without necessarily leading to noticeable bending losses. Therefore, if the cladding glass is less damaged by radiation than the core glass is, the light may propagate in the fibre with an overall lower attenuation when the fibre is bent.

This effect is prominent in the long length sample because the total displacement of the mode field is not instantaneous. The slight displacement of the mode field at the first turn changes the launch conditions of the light at the second turn (hence further displaces the mode field), and so on; this until the mode field reaches its maximum displacement. Therefore the longer the coiled fibre sample is, the greater is the amount of light that gets eventually transferred towards the cladding region.

The fibre investigated is a Ge-doped core fibre, with a pure silica cladding. Pure silica shows a better resistance to radiation than Ge-doped glass [11], which explains that light gets less attenuated when travelling more in the cladding.

The bending influence can be guessed in short length samples as well, since a difference larger than $3.5 \mathrm{~dB} / \mathrm{km}$ could be expected between the $1570 \mathrm{~nm}$ RIA measured at high dose rate and at low dose rate (the difference is of $12 \mathrm{~dB} / \mathrm{km}$ at $1310 \mathrm{~nm}$ ).

\section{FURTHER INVESTIGATION}

Another fibre type was tested for corroboration: a specialty radiation resistant optical fibre (ITU-T G.652.B) whose both core and cladding are doped with Fluorine; the dopant concentration being higher in the cladding than in the core.

According to the fibre manufacturer, 100 turns around a $30 \mathrm{~mm}$ radius spool or 10 turns around a $15 \mathrm{~mm}$ radius spool lead to bending losses that are below $0.05 \mathrm{~dB}$ for both $1310 \mathrm{~nm}$ and $1550 \mathrm{~nm}$. The fibre intrinsic attenuation is of maximum $0.6 \mathrm{~dB} / \mathrm{km}$ at the concerned wavelengths, and its cutoff wavelength is equal to or below $1260 \mathrm{~nm}$.

This fibre type is radiation resistant because the Fluorine dopant decrease the amount of precursor sites (e.g. strained 


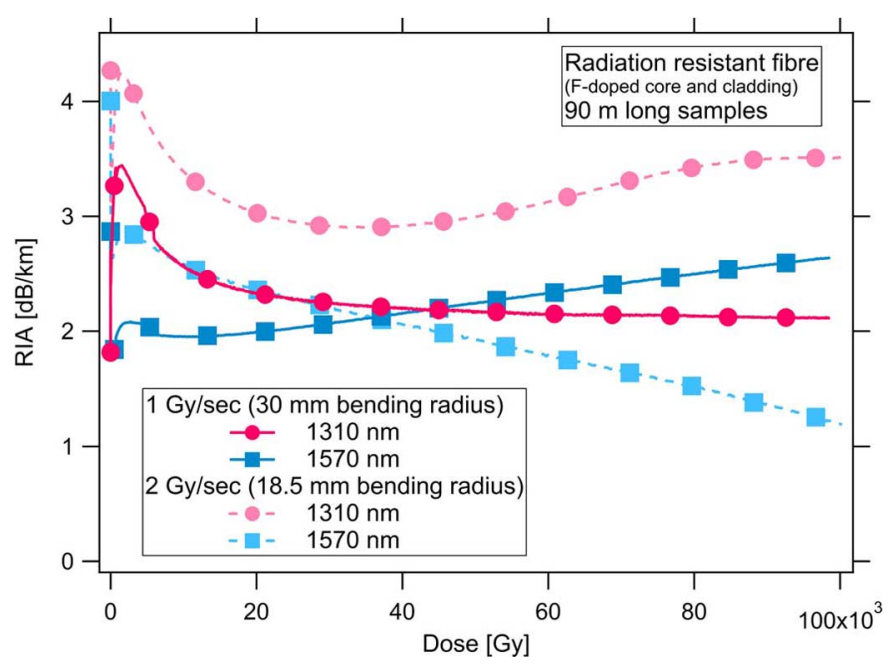

Fig. 4. Radiation resistant fibre (F-doped core and cladding) RIA for two different dose rates and wavelengths.

bonds) that lead to colour centers when irradiated [12]. Fewer $\mathrm{NBOHC}(\equiv \mathrm{Si}-\mathrm{O} \bullet$ ) are then created, thus lowering the formation of silanol ( $\mathrm{Si}-\mathrm{OH}$, -OH absorption band being centered around $1380 \mathrm{~nm}$ ) under irradiation [13][14].

The F-doped fibre irradiation tests were performed with $90 \mathrm{~m}$ length samples exposed $1 \mathrm{~Gy} / \mathrm{s}$ and $2 \mathrm{~Gy} / \mathrm{s}$ dose rates. The results are shown in Fig. 4. The high dose rate (low bending radius) results are shown with dashed lines, whereas the low dose rate (high bending radius) results are shown with solid line. The $1310 \mathrm{~nm}$ results are represented with circle symbols, and the $1570 \mathrm{~nm}$ are represented with square symbols.

As in the telecom standard fibre case (Ge-doped core and pure silica cladding) a higher RIA is expected at $1310 \mathrm{~nm}$ than at $1570 \mathrm{~nm}$ for lower doses, with the RIA at $1570 \mathrm{~nm}$ becoming higher than the RIA at $1310 \mathrm{~nm}$ when the accumulated dose reaches a typical level (crossing point) that varies in function of the dose rate [13]. Moreover, at both wavelengths, the high dose rate tests are expected to show higher RIA than the low dose rate tests [15]. Yet the sample irradiated with a low bending radius and measured at long wavelength (i.e. test conditions $2 \mathrm{~Gy} / \mathrm{s}$, $1570 \mathrm{~nm}$ ) shows an unusual RIA, lower than the RIA observed at lower dose rate and same wavelength. This erratic phenomenon is similar to the one observed in the Ge-doped telecom standard optical fibre. In the range of doping level of the studied fibre $(0.8$ $\mathrm{wt} \%$ for the core and $2.2 \mathrm{wt} \%$ for the cladding), an increase of Fluorine content improves the radiation resistance of the glass [15].

\section{CONCLUSIONS}

The influence of macro-bending on the measured RIA in glass optical fibres is shown to be significant under certain conditions. The measured RIA in long length fibre samples decreases unexpectedly when measured at $1570 \mathrm{~nm}$ with low bending radii, i.e. when bending effects are amplified. This phenomenon is believed to be due to the combination of two factors: the displacement of the mode field towards the cladding region of the bent optical fibre and the difference in colour-centres (types and quantities) created in the core and cladding regions when exposed to radiation.

In both studied Ge-doped telecom standard and F-doped specialty radiation resistant fibres the nature and concentration of the dopants lead to the formation of fewer or of different colourcentres in the cladding region with respect to the core region. Thus, the light travelling in the cladding region is less attenuated, leading to an apparent reduced RIA.

For these reasons, a minimum bending radius should be respected in order to obtain reliable optical tests results. The influence of the bending radius shall be taken into account when measuring the RIA in glass optical fibres in order to prevent RIA underestimation.

For further irradiation tests performed on single-mode optical fibres intended for installation at CERN, a minimum spool radius of $30 \mathrm{~mm}$ is chosen.

\section{REFERENCES}

[1] J. Kuhnhenn, S. K. Höffgen, and U. Weinand, "Quality assurance for irradiation tests of optical fibers: Uncertainty and reproducibility," IEEE Trans. Nucl. Sci., vol. 56, no. 4, pp. 2160-2166, Aug. 2009.

[2] E. J. Friebele and M. E. Gingerich, "Photobleaching effects in optical fiber waveguides," Appl. Opt., vol. 20, no. 19, pp. 3448-3452, Oct. 1981.

[3] D. Marcuse, "Curvature loss formula for optical fibers," J. Opt. Soc. Amer., vol. 66, no. 3, pp. 216-220, Mar. 1976.

[4] D. Marcuse, "Field deformation and loss caused by curvature of optical fibers," J. Opt. Soc. Amer., vol. 66, no. 4, pp. 311-320, Apr. 1976.

[5] M. Young, "Mode-field diameter of single-mode optical fiber by farfield scanning: Addendum," Appl. Opt., vol. 37, no. 36, p. 8361, Dec. 1998.

[6] S. Girard, J. Keurinck, Y. Ouerdane, J. P. Meunier, and A. Boukenter, "Gamma-rays and pulsed X-ray radiation responses of germanosiliscate single-mode optical fibers: Influence of cladding codopants," $J$. Lightw. Technol., vol. 22, no. 8, pp. 1915-1922, Aug. 2004.

[7] D. L. Griscom, M. E. Gingerich, and E. J. Friebele, "Model for the dose, dose-rate and temperature dependence of radiation-induced loss in optical fibers," IEEE Trans. Nucl. Sci., vol. 41, no. 3, pp. 523-527, Jun. 1994.

[8] H. Henschel, O. Kohn, and H. U. Schmidt, "Optical fibres for high radiation dose environments," IEEE Trans. Nucl. Sci., vol. 41, no. 3, pp. 510-516, Jun. 1994.

[9] E. J. Friebele, D. L. Griscom, and G. H. Sigel, "Defects centers in a germanium-doped silica-core optical fiber," J. Appl. Phys., vol. 45, no. 8, pp. 3424-3428, Aug. 1974.

[10] D. L. Griscom, "Optical properties and structure of defects in silica glass," J. Ceramic Soc. Jpn., vol. 99, no. 10, pp. 899-916, Aug. 1991.

[11] S. Girard, J. Keurinck, A. Boukenter, J. P. Meunier, Y. Ouerdane, B. Azaiis, P. Charre, and M. Vié, "Gamma-rays and pulsed X-ray radiation responses if nitrogen-, germanium-doped and pure silica core optical fibers," Nucl. Instrum. Methods Phys. Res. B, vol. 215, pp. 187-195, 2004.

[12] L. Vaccaro, M. Cannas, S. Girard, A. Alessi, A. Morana, A. Boukenter, Y. Ouerdane, and R. Boscaino, "Influence of fluorine on the fiber resistance studied through the nonbridging oxygen hole center related luminescence," J. Appl. Phys., vol. 113, p. 193107, 2013.

[13] T. Wijnands, L. Koert De Jonge, J. Kuhnhenn, S. K. Höffgen, and U. Weinand, "Optical absorption in commercial single mode optical fibers in a high energy physics radiation field," IEEE Trans. Nucl. Sci., vol. 55, no. 4, pp. 2216-2222, Aug. 2008.

[14] T. Wijnands, K. Aikawa, J. Kuhnhenn, D. Ricci, and U. Weinand, "Radiation tolerant optical fibers: From samples testing to large series production," J. Lightw. Technol., vol. 29, no. 22, pp. 3393-3400, Nov. 2011.

[15] K. Aikawa, "Radiation resistant single-mode optical fibers," Fujikura Tech. Rev., no. 37, 2008 [Online]. Available: http://www.fujikura.co.jp/ eng/rd/gihou/backnumber 